\title{
Effect of the stop-signal modality on brain electrical activity associated with suppression of ongoing actions
}

\author{
M.T. Carrillo-de-la-Peña ${ }^{\mathrm{a}, *}$, F.M. Bonilla ${ }^{\mathrm{b}}$, A.J. González-Villar ${ }^{\mathrm{a}, \mathrm{c}}$ \\ ${ }^{a}$ Psychophysiology Lab, Faculty of Psychology, Universdad de Santiago de Compostela, Spain \\ ${ }^{\mathrm{b}}$ Laboratory of Experimental Psychology, Faculty of Psychology, Universidad El Bosque, Colombia \\ ${ }^{c}$ Psychological Neuroscience Lab, Research Center in Psychology, School of Psychology, Universidade do Minho, Portugal
}

\section{A R T I C L E I N F O}

\section{Keywords:}

Motor preparation

Motor inhibition

Stop-signal task

EEG

Stimulus modality effects

Stop-N2/P3

\begin{abstract}
A B S T R A C T
To clarify how the modality of stop signals affects the ability to suppress ongoing actions, we compared behavioural indices and event-related potentials (ERPs) recorded in healthy volunteers performing visual and auditory stop-signal tasks. Auditory stop signals were associated with faster reaction times and shorter stop-N2 and stop-P3 latencies. Given that the tasks did not differ in attentional/arousal processes (go-P3 or stop-P3 amplitudes) or motor preparation (LRP amplitude, onset or latency), our results suggest that stop signal modality mainly affects bottom-up sensory processes (faster auditory processing). The ERP waveform obtained by subtracting successfully stopped from unsuccessfully stopped trials showed similar amplitude and topography in both tasks, indicating that the strength of top-down processes related to inhibition was independent of modality. The findings contribute further knowledge about the variables associated with efficient inhibition and have practical implications for the design of settings or interventions to improve reactive inhibition.
\end{abstract}

\section{Introduction}

Within the field of cognitive neuroscience, there has been a growing interest in inhibitory processes, their related neural systems and their role in cognition (Kok, 1999). Inhibition is the ability to actively suppress, withhold, delay or interrupt ongoing or planned actions, and it is therefore a core executive function (Ridderinkhof, van den Wildenberg, Segalowitz, \& Carter, 2004). Response inhibition can be studied using the go/no-go task and the stop-signal task, which engage overlapping but distinct neural circuits (Swick, Ashley, \& Turken, 2011). As widely recognized, the latter implies greater inhibitory pressure on responserelated processes (Logan, Cowan, \& Davis, 1984; Verbruggen \& Logan, 2008). The ability to cancel already initiated motor responses is crucial in everyday activities (e.g. in driving, avoiding sudden danger...). Study of the variables that modulate performance of the stop-signal task will help to clarify the mechanisms underlying effective inhibition and thus facilitate the design of means to train and improve inhibitory performance.

One of the variables that may affect processes related to effective cancellation of responses is the modality of the stop-signal (Huster, Enriquez-Geppert, Lavallee, Falkenstein, \& Herrmann, 2013). Nevertheless, this variable has not been widely investigated. Most existing studies comparing visual and auditory stop cues have only analyzed behavioural indices and have observed faster stop signal reaction times (SSRT) to auditory stop signals (Morein-Zamir \& Meiran, 2003; van der Schoot, Licht, Horsley, \& Sergeant, 2005). However, the precise locus of this modality-related effect on stopping remains unclear. It has been explained variously by the shorter processing time in the auditory pathway (Elliott, 1968; Goldstone, 1968), the more arousing quality of the tones (van der Schoot et al., 2005) and even as a reflection that go and stop commands are more easily distinguishable in the cross-modality auditory task (Wickens, 1980).

Event-related potentials (ERPs) obtained while performing the stopsignal task provide detailed temporal information on inhibitory processes beyond behavioural data and can help to clarify how functionally distinct processes contribute to the effect of the stop-signal modality. In this vein, Ramautar, Kok, and Ridderinkhof, (2006) observed shorter stop-N2 and stop-P3 latencies and greater modulation of these components in successful stopping vs. unsuccessful stopping in the auditory modality. Given the observed modality-independent topography, the authors concluded that the stop-signal modality primarily affects bottom-up sensory processes and not top-down cognitive processes related to inhibitory control. However, there are some important factors that make it difficult to interpret the ERPs obtained in the stop-signal task. First, the overlap between the EEG activity associated with the perceptual processing of the stop signal and the activity associated with

\footnotetext{
* Corresponding author at: Facultad de Psicologia, Campus Vida, Universidad de Santiago de Compostela, 15702 Santiago de Compsotela, A Coruña, Spain.

E-mail address: mteresa.carrillo@usc.es (M.T. Carrillo-de-la-Peña).
} 
the inhibition process itself reinforces the need to use procedures to separate the two activities. In addition, the authors reported differences in the efficiency of successful stopping for visual and auditory signals that depended on the stop signal delay used. Therefore, given that ERPs are affected by the outcome of the inhibition, we believe that it would be important to ensure a similar percentage of successful/unsuccessful inhibitions in the tasks being compared.

The objective of the present study was to clarify how the stop signal modality affects the different processes involved in the successful cancellation of a response. To this end, we used behavioural data and specific ERP components to disentangle how attention/arousal (P3 amplitudes), timing of inhibitory processes (stop-N2 and stop-P3 latencies), motor response selection and preparation (Lateralized Readiness Potential, LRP) and engagement of inhibitory mechanisms (comparison of ERPs to successful stopping and unsuccessful stopping), contribute to the stop-signal modality effects. To better characterize brain activity during inhibition of visual and auditory signals, we applied a spherical-spline surface Laplacian filter. Use of this type of spatial filter reduces the overlapping between ERPs associated with inhibition (stop-N2 and stop-P3) and those associated with the perceptual processing of the go and stop stimuli. We also used the staircase tracking algorithm to ensure similar rates of successful inhibition in both modalities.

\section{Method}

\subsection{Participants}

Twenty-four students (13 women, 11 men) aged from 20 to 38 years (mean $=22.69 \mathrm{y} / \mathrm{o}$; standard deviation $=4.65)$ participated voluntarily in the study. All participants were right-handed, according to the Edinburgh handedness inventory, and reported having normal or corrected vision. None of them presented a history of neurological or psychiatric disorders, or drug abuse. The EEG recordings from 4 participants were noisy and were therefore excluded from ERP analysis.

\subsection{Task and procedure}

The primary task consisted of a reaction time task to white arrows pointing to the left or the right and indicating which hand to respond with (stimulus duration: $500 \mathrm{~ms}$; mean interstimulus interval: $2 \mathrm{~s}$ ). Each trial began with a fixation target -a plus symbol- presented in the centre of the screen. In the unpredictable, infrequent stop trials, the arrows were followed by a visual or auditory stop signal. Thus, in the visual (same-modality) stop task, a red arrow, pointing left or right, indicated that subjects had to cancel the already prepared response. In the auditory (cross-modality) stop task, an $80 \mathrm{~dB}(1000 \mathrm{~Hz})$ tone was used as the stop signal (see Fig. 1).

During the tasks, participants were seated comfortably in an armchair, in a dimly lit, sound attenuated room. They were instructed to fix their gaze on the plus symbol in the centre of the screen and to respond by pressing a button on a hand-held response box with their left or right thumb according to the direction indicated by the white arrow. In the visual task, the participants were informed that in some trials a red arrow might appear after the white arrow, indicating that the response should be cancelled. In the auditory version, participants were informed that a tone would be the signal to cancel the response. Subjects were instructed to respond as quickly as possible to the white arrow and not to wait for the appearance of the stop signal.

The experiment included some practice trials. The stop tasks were presented in a counterbalanced order: for half of the participants, the order was auditory-visual and for the other half it was visual-auditory. Each task consisted of 280 trials: $70 \%$ were go trials (98 left and 98 right responses) and $30 \%$ were stop trials (42 left, 42 right). The two tasks were separated by a 5 -minute rest interval.

The tasks were designed and presented with the STIM program
(Neuroscan Labs), on a 15 inch screen, at a viewing distance of $100 \mathrm{~cm}$. Reaction times (RTs) and EEG were recorded during performance of the task.

The interval between the onset of the go signal and the stop signal was $300 \mathrm{~ms}$ in the first trial and then varied according to the subject's performance (ranging from 160 to $400 \mathrm{~ms}$ in $40 \mathrm{~ms}$ steps). The variation was achieved using the staircase-tracking algorithm, which adjusts the go-stop interval in a certain trial on the basis of the results of the previous stop trial (Band \& van Boxtel, 1999). This algorithm produces a distribution of around $1 / 2$ of successful and $1 / 2$ of unsuccessful responseinhibited trials. If the response in the previous stop trial was correctly inhibited, the interval between go and stop signals in the next stop trial was $40 \mathrm{~ms}$ longer, also increasing the difficulty of successful inhibition; if the subject responded in the previous stop trial, the interval between signals in the next stop trial was $40 \mathrm{~ms}$ shorter to facilitate inhibition (Logan et al., 1984).

\subsection{Psychophysiological recording and data analysis}

EEGs were recorded from 28 electrode sites (10-20 international system) referenced to the left and right mastoids. The electro-oculogram (EOG) was recorded from sites above and below the left eye and from electrodes lateral to each eye. The AFz electrode served as the ground electrode. Electrode impedance was maintained below $10 \mathrm{k} \Omega$. The EEG signals were digitized online with a Neuroscan equipment (Neuroscan Laboratories, version 4.1), amplified 10,000 x (SynAmp Model 5083 amplifier), filtered online using a band-pass between 0.1 and $100 \mathrm{~Hz}$ and a notch filter of $50 \mathrm{~Hz}$, and sampled at a rate of $500 \mathrm{~Hz}$.

Off-line treatment of the EEG signal (ocular artefact correction, segmentation, $0.1-30 \mathrm{~Hz}$ digital filtering, rejection of noisy epochs, and averaging) was performed with EEGlab software (Delorme \& Makeig, 2004). The EEGs were visually inspected to eliminate segments with noisy recordings and then filtered with a $0.1-30 \mathrm{~Hz}$ band-pass FIR filter. The data were subjected to Independent Component Analysis (ICA), and components identified as ocular or muscular activity were manually rejected. Epochs were extracted from $200 \mathrm{~ms}$ pre-stimulus to $1000 \mathrm{~ms}$ post-stimulus (-200 to $0 \mathrm{~ms}$ baseline correction). Go epochs were extracted time-locked to go stimuli (white arrows) and stop epochs were time-locked to stop stimuli (red arrows or tones). Response-locked epochs were also obtained to compute the lateralized readiness potential (LRP).

To reduce the overlapping between ERPs associated with the inhibitory process (stop-N2 and stop-P3) and with the perceptual processing of go and stop signals, we applied a spherical-spline surface Laplacian filter with the MATLAB code provided by Kayser and Tenke (2006) (smoothing constant $=10^{-5}$ ). This type of filter is useful for removing spatially broad features of data while leaving local features unmodified. It produces sharper topographies of the scalp electrical activity, enhancing the spatial resolution and reducing their overlap due to volume conduction. This transformation provides results scaled to Laplacian units $\left(\mu \mathrm{V} / \mathrm{cm}^{2}\right)$.

In go-locked waveforms, the P3 peak amplitude and latency were measured at the $\mathrm{Pz}$ electrode as the maximum value observed in a window from 250 to $600 \mathrm{~ms}$. As this component had a wide extension in time, and sometimes did not show a clear peak, we also computed the mean amplitude in the aforementioned time window. The ERPs for successfully stopped (SS) and unsuccessfully stopped (US) trials were averaged separately; amplitude and latency were measured at $\mathrm{FCz}$ for stop-N2 (as the minimum value in a window from 100 to $300 \mathrm{~ms}$ ) and at $\mathrm{Cz}$ for stop-P3 (as the maximum value in a window from 180 to $500 \mathrm{~ms}$ ). The time windows and electrodes selected were those that showed the largest amplitude of the components to be analyzed, in accordance with previously established criteria. To further explore ERPs associated with inhibitory processes, we calculated the waveform difference between trials by subtracting the trials that were correctly inhibited (SS) from those where the response was already executed or 
Go Signal

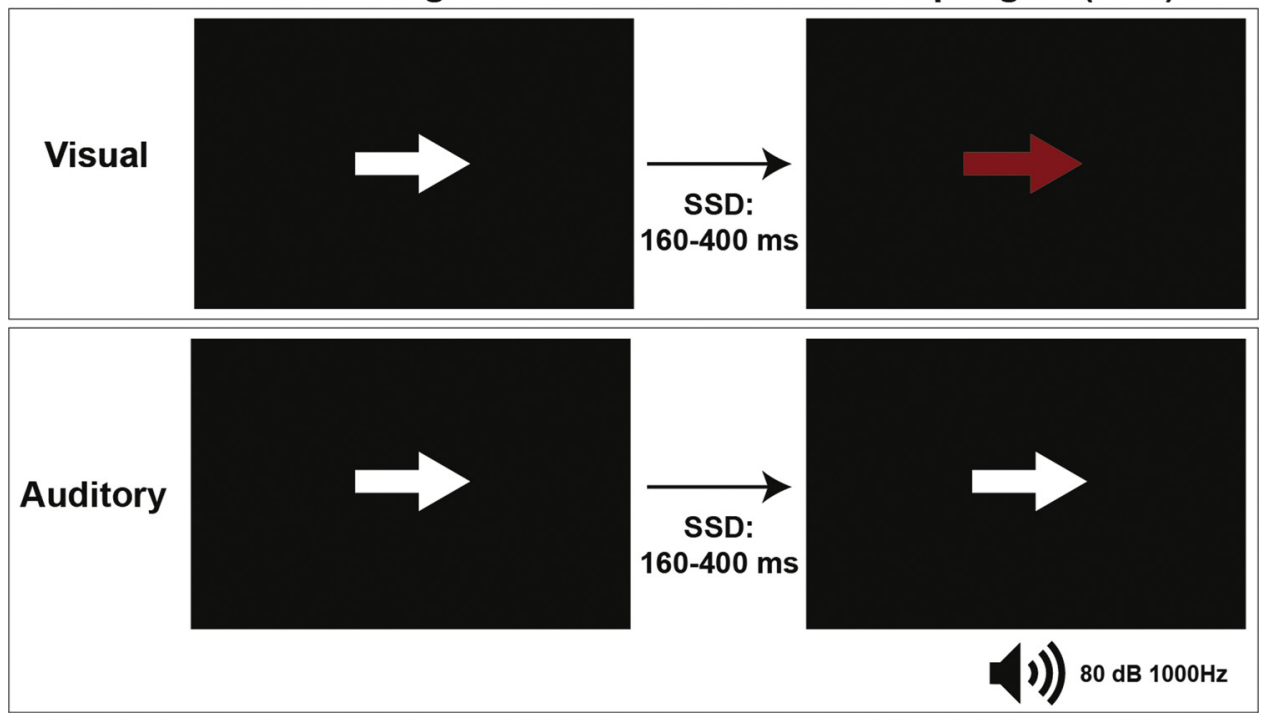

Fig. 1. Task design of visual and auditory stopsignal tasks. Go signals consisted of white arrows pointing to the left or right. Participants had to press a button in a response box with their right or left thumb finger depending on the direction of the arrow. In $30 \%$ of the trials the arrow changed to red (in the visual modality) or a sound was presented (in the auditory modality) indicating that the response (pressing the button) should be cancelled. Arrows lasted $500 \mathrm{~ms}$ and the stop-signal delay (SSD) for stop trials ranged from 160 to $400 \mathrm{~ms}$ (adjusted by the tracking algorithm) (For interpretation of the references to colour in this figure legend, the reader is referred to the web version of this article). not inhibited in time (US), and we measured the peak amplitude and latency in a window from 150 to $320 \mathrm{~ms}$ at $\mathrm{FCz}$ and $\mathrm{Cz}$ electrodes.

The LRP provides information about activation of central preparatory motor processes and has proved to be an adequate measure for locating experimental effects on RTs (Coles, 1989; Eimer, 1998; MüllerGethmann, Ulrich, \& Rinkenauer, 2003; van der Molen, Bashore, Halliday, \& Callaway, 1991). The LRP amplitude indicates the amount of motor preparation in conditions of response execution or cancellation, while LRP onset and latency enable analysis of how a given experimental manipulation affects the reaction times. The LRP can be measured in relation to presentation of the stimulus or to the motor response. Thus, we calculated the go stimulus-locked lateralized readiness potential (S-LRP) separately for go, SS and US trials, and the response-locked lateralized readiness potential (R-LRP) for go and US trials. LRP computation was made using the average method proposed by Coles (1989) (i.e. by calculating C3 minus C4 for right-hand responses and C4 minus C3 for left-hand responses, and then averaging the resulting ERP difference waveforms). As the LRP should not be affected by other stimulus-evoked ERPs, such as N1 or P1, it was computed without applying the Laplacian transformation.

The following statistical analyses were subsequently applied to performance- and ERP-related measures. Descriptive analysis and dependent sample t-tests (visual vs. auditory) were performed for all behavioural indices obtained, from go (\% hits, \% errors, \% missing, RTs for hits and for errors) and stop trials (\% US, \% SS, RTs in US, stopsignal delay [SSD], and stop-signal reaction time [SSRT]). The SSRTs represent the point at which the stopping process finishes and can be estimated by taking into account the go RT distribution and the observed probability of successful/unsuccessful inhibitions to the stop signal for a given SSD. The SSRT was computed by subtracting the observed mean SSD from the mean go RT (Logan et al., 1984; Logan, Schachar, \& Tannock, 1997).

Concerning electrophysiological data, dependent-sample t-tests (visual vs. auditory) were applied for go-P3 amplitude and latency. Repeated measures ANOVAs (with Modality x Performance, i.e. US vs. SS) were performed for stop-N2 and stop-P3 peak latency and amplitude, and also for the S-LRP onset and amplitude (go, US and SS) and RLRP onset (go and US) as dependent variables. Post-hoc comparisons were Bonferroni corrected.

\section{Results}

\subsection{Behavioural performance}

Behavioural indices for go and stop trials separating visual and auditory stop-signal tasks (mean of right- and left- responses) are shown in Table 1.

Student's t-tests showed significant modality effects for go-RTs $\left(\mathrm{t}_{(23)}=-2.17 ; \mathrm{p}=.041\right)$, and SSRTs $\left(\mathrm{t}_{(23)}=-2.33 ; \mathrm{p}=.029\right)$. The posthoc comparison showed shorter RTs in the auditory than in the visual modality, for both go and stop trials. RTs to unsuccessfully stopped trials were also larger for visual stop-signals, although the effect was not significant $\left(\mathrm{t}_{(23)}=-1.89 ; \mathrm{p}=.071\right)$. As an expected consequence of the use of the staircase-tracking algorithm, no significant differences in the rate of successful vs successful inhibitions were found between the auditory and visual stop tasks. Similarly, modality did not significantly affect the percentage of hits in the primary task (go responses).

\subsection{ERPs}

\subsubsection{Go $P 3$}

Data for all the ERP indices are presented in Table 2. The modality effect was not significant for P3 peak amplitude $\left(\mathrm{t}_{(19)}=1.55 ; \mathrm{p}=.14\right)$ or peak latency $\left(\mathrm{t}_{(19)}=0.2 ; \mathrm{p}=.8\right)$, nor for P3 mean amplitude as the

Table 1

Behavioural results for each condition. Standard deviations are shown in parentheses.

\begin{tabular}{lll}
\hline & Auditory & Visual \\
\hline Go trials & & \\
\% Hits & $95.77(3.60)$ & $94.64(6.74)$ \\
\% Errors & $1.81(1.85)$ & $2.40(2.79)$ \\
\% Misses & $2.42(3.42)$ & $2.95(5.81)$ \\
RTs for Hits & $413.06(61.26)$ & $438.15(60.35)$ \\
RTs for Errors & $237.72(43.45)$ & $309.32(93.50)$ \\
Stop trials & & \\
\% US & $47.27(14.84)$ & $53.22(17.45)$ \\
\% SS & $52.73(14.84)$ & $46.78(17.45)$ \\
RTs for US & $372.13(44.11)$ & $389.54(41.86)$ \\
SSD & $239.23(31.98)$ & $247.06(45.81)$ \\
SSRT & $173.83(37.10)$ & $191.09(27.98)$ \\
\hline
\end{tabular}

Abbreviations. RT: Reaction time; US: Unsuccessful stopping; SS: Successful stopping; SSD: Stop-signal delay; SSRT: Stop-signal reaction time. RTs, SSD and SSRT are reported in ms. 
Table 2

Mean amplitudes (in $\mu \mathrm{V} / \mathrm{cm}^{2}$, except for LRP peaks, with amplitudes shown in $\mu \mathrm{V}$ ) and latencies (in ms) for each of the components analyzed. Standard deviations are shown in parentheses.

\begin{tabular}{|c|c|c|}
\hline & Auditory & Visual \\
\hline \multicolumn{3}{|l|}{ Go P3 (Pz) } \\
\hline Peak amplitude & $0.31(0.14)$ & $0.28(0.14)$ \\
\hline Peak latency & $326(38)$ & $323(45)$ \\
\hline Mean amplitude & $0.19(0.11)$ & $0.18(0.12)$ \\
\hline \multicolumn{3}{|l|}{ Stop-N2 (FCz) } \\
\hline SS amplitude & $-0.42(0.27)$ & $-0.17(0.16)$ \\
\hline SS latency & $144(18)$ & $224(52)$ \\
\hline US amplitude & $-0.48(0.31)$ & $-0.37(0.24)$ \\
\hline US latency & $150(14)$ & $250(25)$ \\
\hline \multicolumn{3}{|l|}{ Stop-P3 (Cz) } \\
\hline SS amplitude & $0.53(0.39)$ & $0.43(0.23)$ \\
\hline SS latency & $247(47)$ & $304(71)$ \\
\hline US amplitude & $0.37(0.27)$ & $0.37(0.22)$ \\
\hline US latency & $268(52)$ & $340(90)$ \\
\hline \multicolumn{3}{|l|}{ US-SS difference } \\
\hline Peak amp FCz & $-0.33(0.19)$ & $-0.32(0.19)$ \\
\hline Peak lat FCz & $207(39)$ & $270(29)$ \\
\hline Peak amp Cz & $-0.36(0.27)$ & $-0.33(0.28)$ \\
\hline Peak lat Cz & $210(41)$ & $261(29)$ \\
\hline \multicolumn{3}{|l|}{ S-LRP } \\
\hline Peak amp Go & $-1.21(0.65)$ & $-1.09(0.58)$ \\
\hline Peak lat Go & $335(72)$ & $352(65)$ \\
\hline Onset lat Go & $166(40)$ & $175(47)$ \\
\hline Peak amp US & $-1.53(0.70)$ & $-1.52(0.88)$ \\
\hline Peak lat US & $313(55)$ & $358(61)$ \\
\hline Onset lat US & $197(41)$ & $208(41)$ \\
\hline Peak amp SS & $-1.03(0.64)$ & $-1.17(0.63)$ \\
\hline Peak lat SS & $348(70)$ & $349(71)$ \\
\hline Onset lat SS & $199(44)$ & 204(49) \\
\hline \multicolumn{3}{|l|}{ R-LRP } \\
\hline Peak amp Go & $-1.25(0.70)$ & $-1.34(0.69)$ \\
\hline Peak lat Go & $-22(48)$ & $-14(62)$ \\
\hline Onset lat Go & $-195(63)$ & $-190(61)$ \\
\hline Peak amp US & $-1.58(0.83)$ & $-1.44(0.73)$ \\
\hline Peak lat US & $-17(80)$ & $-17(71)$ \\
\hline Onset lat US & $-180(72)$ & $-177(58)$ \\
\hline
\end{tabular}

Abbreviations. lat: latency; amp: amplitude; US: Unsuccessful stopping; SS: Successful stopping; S-LRP: stimulus-locked lateralized readiness potential; RLRP: response-locked lateralized readiness potential.

\section{ERPs for go trials}

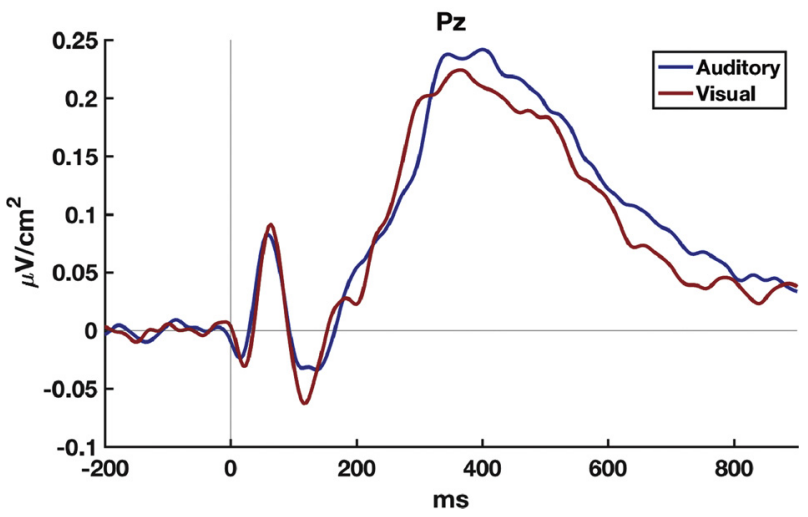

Fig. 2. Event-related potential (ERP) for go trials at the Pz electrode.

mean value from 250 to $600 \mathrm{~ms}\left(\mathrm{t}_{(19)}=0.6 ; \mathrm{p}=.5\right)$, all measured at the Pz electrode (see Fig. 2).

\subsubsection{Stop-N2}

Using the stop-N2 peak amplitude at FCz as the dependent variable, we found significant effects for Modality (larger amplitudes for the auditory than for visual modality; $\mathrm{F}_{(1,19)}=18.2 ; \mathrm{p}<.001$ ) and
Performance (larger amplitudes for US than SS trials; $F_{(1,19)}=16.6$; $\mathrm{p}=.001$ ), and for the Modality $\mathrm{x}$ Performance interaction $\left(\mathrm{F}_{(1,19)}=15.3 ; \mathrm{p}=.001\right)$. Post-hoc comparisons revealed larger stopN2 peak amplitude for US than SS trials, but only in the visual modality $\left(\mathrm{t}_{(19)}=5.6 ; \mathrm{p}<.001\right)$.

For the stop-N2 latency, we found slower latencies for the visual than the auditory modality $\left.\left(\mathrm{F}_{(} 1,19\right)=191.7 ; \mathrm{p}<.001\right)$, and for US than SS trials $\left.\left(\mathrm{F}_{(} 1,19\right)=9.3 ; \mathrm{p}=.007\right)$, but no interaction between modality and performance $\left(\mathrm{F}_{(} 1,19\right)=3.9 ; \mathrm{p}=.06$ ) (see Fig. 3 , top left).

\subsubsection{Stop-P3}

For stop-P3 peak amplitude at the $\mathrm{Cz}$ electrode, the ANOVA revealed significant effects for Performance $\left.\left(\mathrm{F}_{(} 1,19\right)=12.2 ; \mathrm{p}=.002\right)$, with larger amplitudes for SS trials, and for the Modality x Performance interaction $\left.\left(\mathrm{F}_{(} 1,19\right)=8.2 ; \mathrm{p}=.01\right)$. Post-hoc comparisons showed that stop-P3 differences between US and SS trials only appeared in the auditory modality $\left(\mathrm{t}_{(19)}=3.9 ; \mathrm{p}=.001\right)$. The effect of Modality was not significant $\left.\left(\mathrm{F}_{(} 1,19\right)=0.6 ; \mathrm{p}=.4\right)$.

For the stop-P3 latency, significant effects were observed for Modality $\left.\left(\mathrm{F}_{(} 1,19\right)=10.1 ; \mathrm{p}=.005\right)$, with slower latencies in the visual task, and Performance $\left.\left(\mathrm{F}_{(} 1,19\right)=11.5 ; \mathrm{p}=.003\right)$, with slower latencies for US trials. No Modality x Performance interaction was observed $\left(\mathrm{F}_{(1,19)}=0.5 ; \mathrm{p}=.5\right)$ (see Figure 3 , top right).

\subsubsection{US-SS difference}

The waveforms resulting by subtracting ERPs associated with SS trials from those associated with US trials showed that at FCz, the peak has earlier latency in the auditory modality $\left(\mathrm{t}_{(19)}=-5.12 ; \mathrm{p}<.001\right)$, with no difference in amplitude across modalities $\left(\mathrm{t}_{(19)}=-0.48\right.$; $\mathrm{p}=.64$ ). Similar results were found for the $\mathrm{Cz}$ electrode, with modality differences in latency $\left(\mathrm{t}_{(19)}=-5.06 ; \mathrm{p}<.001\right)$ but not in amplitude $\left(\mathrm{t}_{(19)}=-0.75 ; \mathrm{p}=.46\right)$. A similar fronto-central topography was observed in both modalities for this difference waveform (see Figure 3, bottom)

\subsubsection{Stimulus-locked lateralized readiness potential (S-LRP)}

Data on peak latency, amplitude and onset for LRP obtained from go stimuli-locked EEG trials are also shown in Table 2. For S-LRP amplitudes, the repeated measures ANOVA showed a main effect of type of trial $\left.\left(\mathrm{F}_{(} 2,38\right)=9.83 ; \mathrm{p}<.001\right)$. Post-hoc comparisons showed that US $(x=-1.53)$ presented significantly larger amplitude than go $(x=-1.15$; $\mathrm{p}<.001)$ and SS trials $(\mathrm{x}=-1.10 ; \mathrm{p}<.05)$, irrespective of task modality. No significant effects of type of trial were observed for S-LRP peak latency. For S-LRP onset, the effect of type of trial was significant $\left.\left(F_{2} 2,38\right)=6.57 ; \mathrm{p}=.004\right)$, with faster onset times for $\mathrm{Go}(170 \mathrm{~ms})$ than for the other conditions (US $=202 \mathrm{~ms}, \mathrm{p}=.021$; $S S=201 \mathrm{~ms}$, $\mathrm{p}=.014)$. The effect of Modality was not significant in any of the ANOVAs.

\subsubsection{Response-locked lateralized readiness potential ( $R-L R P$ )}

No significant effect was found for R-LRP amplitude, onset or latency (see Figure 4).

\section{Discussion}

In this study, we investigated how the stop signal modality affects inhibitory performance and brain activity, by comparing ERPs associated with stimuli processing time, attention/arousal, motor preparation and engagement of inhibitory processes in auditory vs. visual stopsignal tasks. Both reaction times and ERP latencies demonstrated faster inhibition to auditory cues, while the various indices of attention/ arousal, motor preparation or engagement of inhibitory processes did not significantly differentiate between modalities.

First, the findings showed that the auditory modality was associated with faster stop-signal reaction times (SSRTs), as well as with shorter stop-N2 and stop-P3 latencies. The difference in the speed of stopping 


\section{ERPs for stop trials}
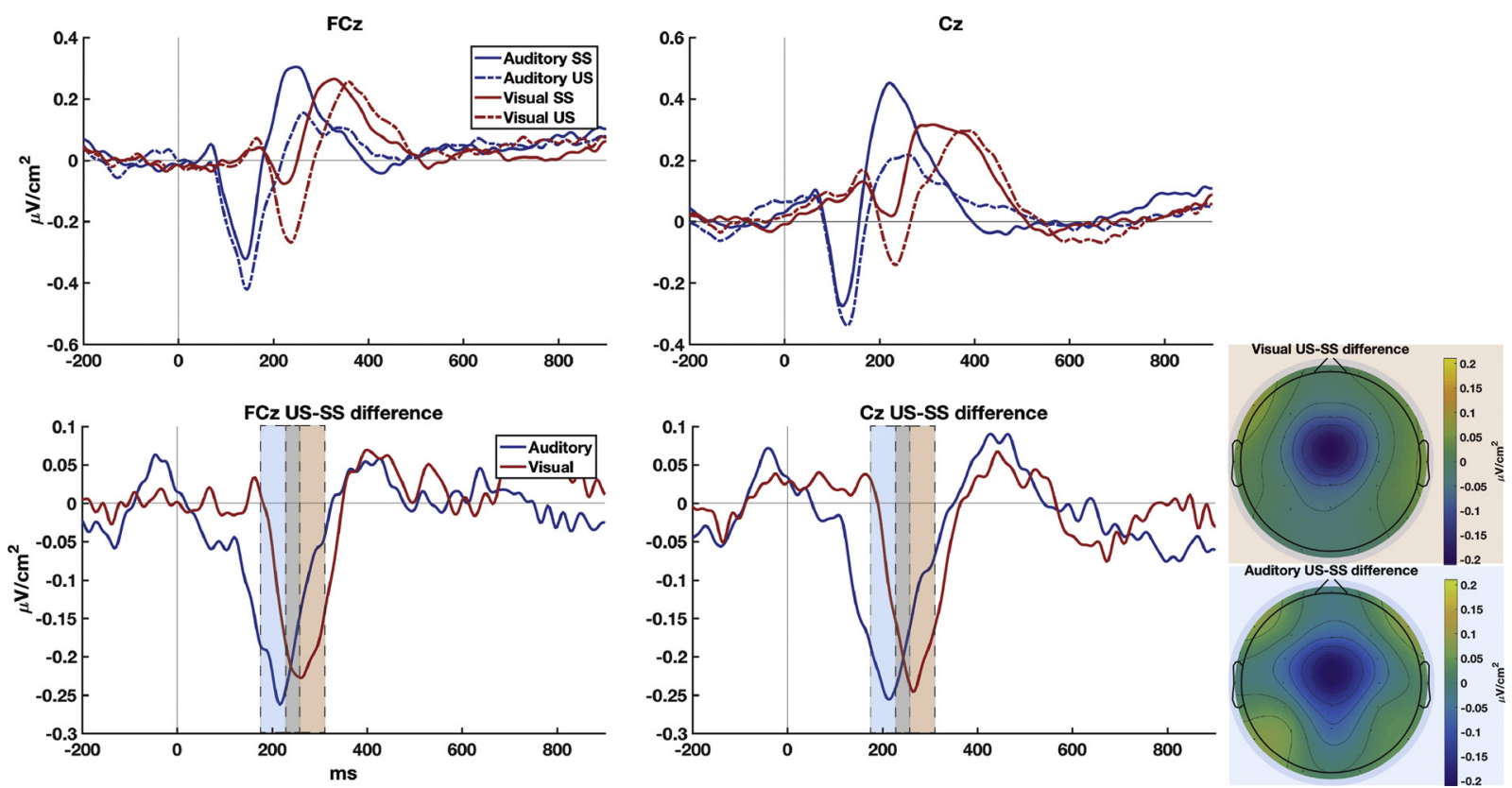

Fig. 3. Top: Event-related potentials (ERPs) -locked to the presentation of the stop signal- at the FCz and Cz electrodes for successfully stopped (SS) and unsuccessfully stopped (US) trials. Bottom: US-SS difference at FCz and $\mathrm{Cz}$, and the topographies for each modality. Shaded areas represent the time windows used for calculating the topographies in each modality.

between the auditory and visual stop tasks was previously observed and explained in terms of the shorter neural pathway for auditory stimulation, with fewer synapses (Elliott, 1968; Goldstone, 1968). Alternatively, the faster response to and inhibition times for the sounds have also been explained in terms of the greater arousal/attentional engagement for auditory than visual stop signals, as proposed by van der Schoot et al. (2005). These authors compared auditory stop stimuli of $80 \mathrm{~dB}$ and $60 \mathrm{~dB}$ and found that only the former speeded the executive processes of inhibition, suggesting that the physical quality of the stimuli affects top-down inhibitory processes. However, our results regarding the amplitude of the P3 component do not seem to support this interpretation in terms of the greater capacity to arouse or engage attentional resources of the auditory stop cues. If this were the case, we would observe larger amplitudes of go-P3, an index of the amount of attentional resources engaged in the processing of the stimuli, in the auditory than in the visual stop-signal task. Nevertheless, we did not find any significant difference between modalities for go-P3.

On the other hand, we did not observe any modality effects for stopP3 amplitude, a fronto-cental positivity considered a neural signature of inhibitory control (Wessel \& Aron, 2015; Wessel, 2017). In go/no-go tasks, the no-go P3 amplitude has been related to suppression of an overt motor response, as it is larger in situations requiring response suppression or change, more particularly as the inhibition is made more demanding (Kropotov, Ponomarev, Hollup, \& Mueller, 2011; Randall \& Smith, 2011; Smith, Johnstone, \& Barry, 2007). Similarly, the stop-P3 amplitude is larger in fast than in slow responses (Dimoska, Johnstone, \& Barry, 2006; Ramautar et al., 2006). The lack of modality differences in stop-P3 amplitude does not support the idea that inhibition processes are more strongly involved in auditory stop-signal tasks. Some authors argue that P3 occurs too late to correspond to an actual motor inhibition process and that the peak of this component may be the result of earlier neural events. Huster et al. (2013) suggested that stop-P3 may be an after-effect of inhibition, possibly involving evaluation of the inhibitory performance, while Wessel and Aron (2015) proposed using the onset of P3 as a neural marker of the response inhibition process. Our findings indicate that inhibition and/or its evaluation are quicker when auditory stop signals are used, but the time delay observed in the visual modality does not seem to be due to weaker involvement of inhibitory processes.

To better understand the effects of inhibition outcome, we analyzed the difference between ERPs elicited in response to unsuccessfully (US) and successfully stopped trials (SS). The waveforms obtained showed a negative potential with fronto-central topography for both auditory and visual stop-signal tasks (Fig. 2 Fig. 3). Again, no modality differences for the amplitude of the US-SS difference were observed. Thus, we can conclude that top-down inhibition mechanisms are activated with similar intensity and topography after both visual and auditory stopsignals. This conclusion is consistent with that reached by Ramautar et al (2006). On the basis of the similar topography found for stop-N2 and stop-P3, these authors concluded that stop-signal modality only affects bottom-up processes.

It could be argued that the differences in the speed of stopping between the auditory and visual stop tasks may result from differences in excitability associated with motor response selection and execution processes. To clarify this aspect, we calculated the lateralized readiness potential (LRP). The LRP is mainly related to the activation of primary motor cortex (Böcker, Brunia, \& Cluitmans, 1994) and its amplitude provides information about the amount of motor preparation associated with response execution or cancellation (van Boxtel, van der Molen, Jennings, \& Brunia, 2001). The lack of differences in LRP amplitude across modalities does not explain the faster inhibition in the auditory modality in terms of motor preparation. LRP amplitudes were larger for failed stop trials than for go and successfully stopped trials, irrespective of the stop-signal modality. As observed in previous studies, the larger LRP amplitude in US trials demonstrates that uninhibited responses show increased engagement of motor preparation processes than the correctly inhibited ones (van Boxtel et al., 2001).

As far as we are aware, there are no previous reports of the effects of the stop-signal modality on LRP latency. To fully understand the contribution of motor preparation and execution processes to the differences between auditory and visual stop-signal tasks, we calculated LRP locked to the go stimulus (S-LRP) and LRP locked to the overt response 


\section{Stim-Locked LRPs}
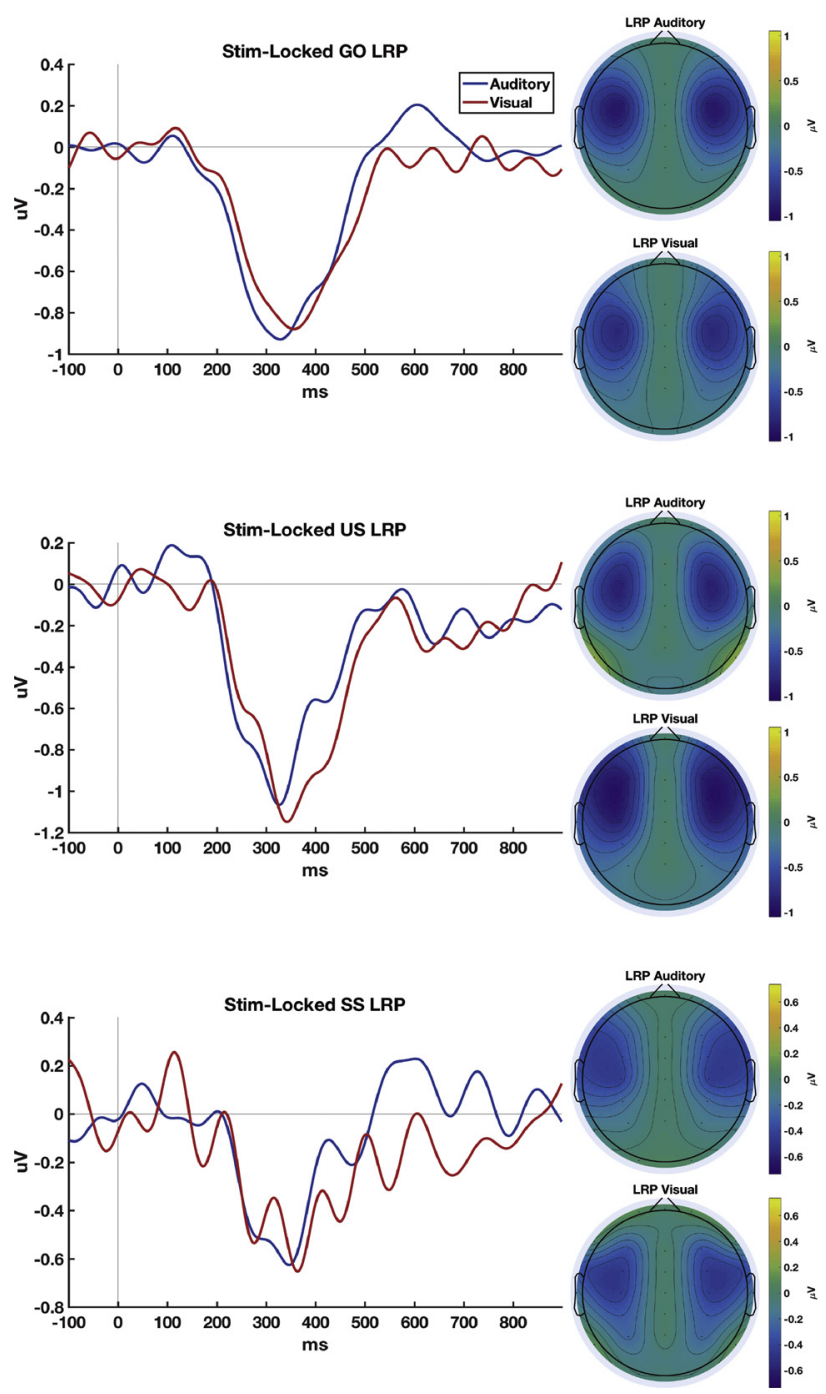

Response-Locked LRPs
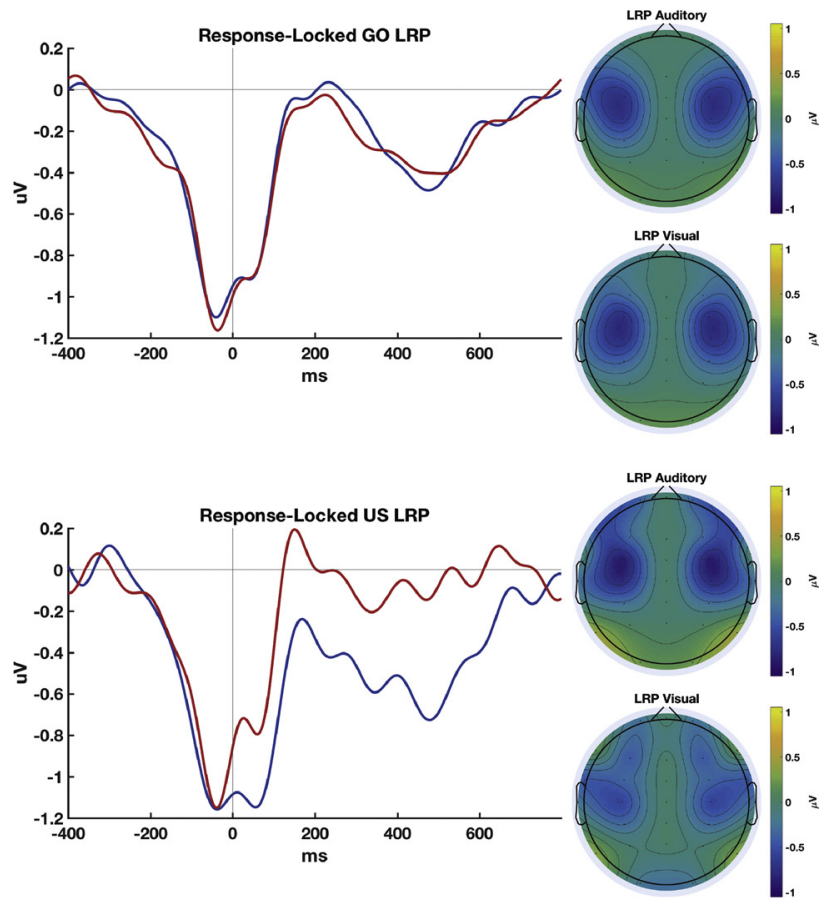

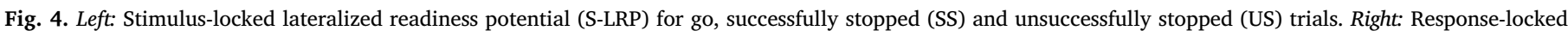

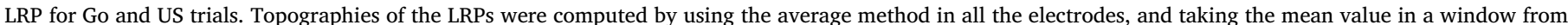
$250 \mathrm{~ms}$ to $400 \mathrm{~ms}$ for stimulus-locked LRPs and from $-100 \mathrm{~ms}$ to $150 \mathrm{~ms}$ for response-locked LRPs.

(R-LRP); we did not find any significant modality effect for either of the temporal parameters (peak or onset latency). Thus, we can conclude that the auditory cues did not affect motor processes at early (from the presentation of the stimuli to the beginning of hand response activation) or late phases (from the beginning of hand response activation to the emission of the motor response) and that motor preparatory processes do not account for the modality differences found in ERPs latencies.

The findings also have theoretical implications for the study of stopsignal tasks and for the interpretation of stop-N2 and stop-P3 components.

First, as also reported by Ramautar et al. (2006), we found faster RTs for go trials in the auditory than in the visual task (note that in those trials there were no stop tones, and that go signals consisted of white arrows pointing to the right or left in both tasks). This finding challenges the foundations of the horse-race model, which predicts complete independence between the stop and the go processes (Verbruggen \& Logan, 2009). Given that stop-signal modality affects go RTs, our data showed that operations related to the go response are not completely independent of the response to stop signals. To explain this result, we propose that the presence of auditory cues may alter the strategy of the participants while performing the task: as the auditory stop signal is processed faster, the waiting time during which the participant speculates on the appearance of the stop signal is reduced and the response is executed more quickly.

Second, our results reinforce the need to use EEG analysis procedures to separate brain activity linked to inhibitory processes from those linked to stimulus processing, as the overlapping between both activities may explain some of the inconsistencies found in the literature. Unlike Ramautar et al. (2006), we found larger stop-N2 peak amplitude in the auditory stop-signal task. A closer look at the data of Ramautar et al. (2006) shows that in the auditory task, the N2 is superimposed on the rising flank of a large P2, which may cause the positive polarity of auditory stop-N2 found by those authors. In the present study, we applied spherical-spline surface Laplacian filtering to reduce the overlapping between ERP components. Nevertheless, given the proximity (in temporal and topographical terms) of the auditory N1-P2 complex and the inhibitory stop-N2, this component should be interpreted carefully.

Our results recommend caution in the interpretation of stop-N2 and stop-P3. The stop-N2 amplitude has been interpreted as an index of conflict monitoring (Botvinick, Cohen, \& Carter, 2004; Carter et al., 
1998). In line with previous reports (Dimoska et al., 2006; EnriquezGeppert, Konrad, Pantev, \& Huster, 2010), our results confirmed larger stop-N2 amplitudes for US than SS trials, but only for the visual task. This finding is consistent with the predictions of the horse race model, i.e. that the conflict introduced by the stop signal may be larger for failed inhibitions (stop signal appears when the go responses are near execution) than for easily inhibited responses. Regarding stop-P3, and also as previously reported (Dimoska, Johnstone, Barry, \& Clarke, 2003), we found larger amplitudes for SS than for US trials (indicating larger engagement of inhibitory processes in successful inhibition), but only in the auditory tasks. Analysis of the waveforms obtained by subtracting SS from US trials help us to understand the discrepant results for each modality task. In the visual modality, the US-SS difference had maximal amplitude at a point temporarily closer to stop-N2, while in auditory modality the difference was maximal at a point closer to stop-P3. Thus, our data suggest that the inhibitory processes triggered by the stop signal cannot be broken down into conflict detection and inhibition itself, i.e. they suggest that stop-N2 and stop-P3 seem to be correlates of a unique process, which, depending on the modality, may present a waveform peak sooner or later. The finding highlights the difficulty in interpreting these components and suggests that they are affected differently depending on the speed at which the stimulus is processed.

Finally, the data suggest some similarity between the US-SS difference waveform and ERP components related to error processing. Given the topography, latency and polarity, the activity is probably associated with the error-related negativity (ERN), a negative component that appears around 50-60 ms after an erroneous response and presumably represents the detection of a mismatch between the correct response and the performed response (Coles, Scheffers, \& Holroyd, 2001; Falkenstein, Hoormann, Christ, \& Hohnsbein, 2000; Gehring, Goss, Coles, Meyer, \& Donchin, 1993). Thanks to the tracking algorithm used, the ratio of US/SS trials was similar in both modalities, and thus the findings should not be affected by probability effects or the rate of successful stopping.

This study also allows us to glimpse some lines of future research. It has been suggested that faster RTs in the auditory task may reflect that go and stop commands are more easily distinguishable in the crossmodality auditory task (Wickens, 1980). It would be interesting to use a single-modality auditory task with different sounds for go and stop signals, and also to explore the effect of other stimuli (somatosensory, haptic, emotional) as stop cues. In this vein, several recent studies have manipulated the emotional or rewarding value of stop cues, showing that threatening arousing stimuli and unpleasant sounds facilitate behavioral inhibition and error processing (Senderecka, 2016, 2017). Rewarding stop was also associated with faster SSRT and larger P3 amplitude difference between successful versus failed inhibitions, suggesting increased attentional processing to the rewarding trials (Greenhouse and Wessel, 2013; Schevernels et al., 2015).

Our findings have some practical implications. In a recent study, Wessel and Aron (2014) demonstrated that simple stop-signal tasks and complex stopping tasks activated the same brain network. Thus, research into the effects of stop-signal modality can help to better identify the variables involved in efficient stopping in more realistic settings. As a practical example in the field of road safety, it is possible that inhibition would occur faster if conventional visual stop signals (vertical signals, road signaling, traffic lights) incorporated some auditory elements (noisy vibrations, beeping, etc.). Information obtained from ERPs obtained in stop-trial tasks could also be used to design training strategies to improve inhibitory processes in populations with dysfunctional inhibitory control (as impulsive young offenders or obsessivecompulsive patients). As shown in previous reports, the inhibitory network is also activated during the covert execution of the stop-signal task (González-Villar, Bonilla, \& Carrillo-de-la-Peña, 2016; GaldoÁlvarez, Bonilla, González-Villar, \& Carrillo-de-la-Peña, 2016), and it would thus also be possible to covertly train more efficient stopping strategies.

\section{Conclusion}

The modality differences in behavioural and ERP latencies obtained during the performance of a reactive inhibitory task confirmed that auditory stop signals are associated with faster cancellation of responses. Analysis of specific ERP components enabled us to clarify the contribution of processes associated with processing time, attention/ arousal, motor preparation and inhibition to the differences observed between auditory and visual stop-signal tasks. Results for P3 amplitude, US-SS difference waveform and the LRP suggest that the stop-signal modality does not affect top-down processes related to inhibition, error processing or motor preparation. The findings question previous theoretical interpretations of stop-N2 and stop-P3 and also reinforce the need to use procedures to separate brain activity linked to inhibition from that linked to go and stop signals processing. They also have implications for the design of settings and interventions to facilitate faster inhibition of already initiated responses.

\section{Acknowledgements}

This work was supported by funding from the Galician Government (Consellería de Cultura, Educación e Ordenación Universitaria; axudas para a consolidación e Estruturación de unidades de investigación competitivas do Sistema universitario de Galicia [grant number GPC2014/047]. A.J. G-V was supported by a grant from the Deputación da Coruña (Bolsas de investigación en ciencias da saúde 2017) and Xunta de Galicia (Axudas de apoio á etapa de formación posdoutoral 2018).

\section{References}

Band, G. P., \& van Boxtel, G. J. (1999). Inhibitory motor control in stop paradigms: Review and reinterpretation of neural mechanisms. Acta Psychologica, 101(2-3), 179-211. Retrieved from http://www.ncbi.nlm.nih.gov/pubmed/10344185.

Böcker, K. B. E., Brunia, C. H. M., \& Cluitmans, P. J. M. (1994). A spatio-temporal dipole model of the readiness potential in humans. I. Finger movement.

Electroencephalography and Clinical Neurophysiology, 91(4), 275-285. https://doi.org/ 10.1016/0013-4694(94)90191-0.

Botvinick, M. M., Cohen, J. D., \& Carter, C. S. (2004). Conflict monitoring and anterior cingulate cortex: An update. Trends in Cognitive Sciences, 8(12), 539-546. https://doi. org/10.1016/j.tics.2004.10.003.

Carter, C. S., Braver, T. S., Barch, D. M., Botvinick, M. M., Noll, D., \& Cohen, J. D. (1998) Anterior cingulate cortex, error detection, and the online monitoring of performance. Science (New York, N.Y.), 280(5364), 747-749. Retrieved from http://www.ncbi.nlm. nih.gov/pubmed/9563953.

Coles, M. G. (1989). Modern mind-brain reading: Psychophysiology, physiology, and cognition. Psychophysiology, 26(3), 251-269. Retrieved from http://www.ncbi.nlm. nih.gov/pubmed/2667018.

Coles, M. G., Scheffers, M. K., \& Holroyd, C. B. (2001). Why is there an ERN/Ne on correct trials? Response representations, stimulus-related components, and the theory of error-processing. Biological Psychology, 56(3), 173-189. Retrieved from http://www. ncbi.nlm.nih.gov/pubmed/11399349.

Delorme, A., \& Makeig, S. (2004). EEGLAB: An open source toolbox for analysis of singletrial EEG dynamics including independent component analysis. Journal of Neuroscience Methods, 134(1), 9-21. https://doi.org/10.1016/j.jneumeth.2003.10. 009.

Dimoska, A., Johnstone, S. J., Barry, R. J., \& Clarke, A. R. (2003). Inhibitory motor control in children with attention-deficit/hyperactivity disorder: Event-related potentials in the stop-signal paradigm. Biological Psychiatry, 54(12), 1345-1354.

Dimoska, A., Johnstone, S. J., \& Barry, R. J. (2006). The auditory-evoked N2 and P3 components in the stop-signal task: Indices of inhibition, response-conflict or errordetection? Brain and Cognition, 62(2), 98-112. https://doi.org/10.1016/j.bandc. 2006.03.011.

Eimer, M. (1998). The lateralized readiness potential as an on-line measure of central response activation processes. Behavior Research Methods Instruments \& Computers, 30(1), 146-156. https://doi.org/10.3758/BF03209424.

Elliott, R. (1968). Simple visual and simple auditory reaction time: A comparison. Psychonomic Science, 10(10), 335-336. https://doi.org/10.3758/BF03331548.

Enriquez-Geppert, S., Konrad, C., Pantev, C., \& Huster, R. J. (2010). Conflict and inhibition differentially affect the N200/P300 complex in a combined go/nogo and stop-signal task. NeuroImage, 51(2), 877-887. https://doi.org/10.1016/j. neuroimage.2010.02.043.

Falkenstein, M., Hoormann, J., Christ, S., \& Hohnsbein, J. (2000). ERP components on 
reaction errors and their functional significance: A tutorial. Biological Psychology, 51(2-3), 87-107. https://doi.org/10.1016/S0301-0511(99)00031-9.

Galdo-Álvarez, S., Bonilla, F. M., González-Villar, A. J., \& Carrillo-de-la-Peña, M. T. (2016). Functional equivalence of imagined vs. real performance of an inhibitory task: An EEG/ERP study. Frontiers in Human Neuroscience, 10, 467.

Gehring, W. J., Goss, B., Coles, M. G. H., Meyer, D. E., \& Donchin, E. (1993). A neural system for error detection and compensation. Psychological Science, 4(6), 385-390. https://doi.org/10.1111/j.1467-9280.1993.tb00586.x.

Goldstone, S. (1968). Reaction time to onset and termination of lights and sounds. Perceptual and Motor Skills, 27(3_suppl), 1023-1029. https://doi.org/10.2466/pms. 1968.27.3f.1023.

González-Villar, A. J., Bonilla, F. M., \& Carrillo-de-la-Peña, M. T. (2016). When the brain simulates stopping: neural activity recorded during real and imagined stop-signal tasks. Cognitive, Affective, \& Behavioral Neuroscience, 16(5), 825-835.

Greenhouse, I., \& Wessel, J. R. (2013). EEG signatures associated with stopping are sensitive to preparation. Psychophysiology, 50(9), 900-908.

Huster, R. J., Enriquez-Geppert, S., Lavallee, C. F., Falkenstein, M., \& Herrmann, C. S. (2013). Electroencephalography of response inhibition tasks: Functional networks and cognitive contributions. International Journal of Psychophysiology, 87(3), 217-233. https://doi.org/10.1016/j.ijpsycho.2012.08.001.

Kayser, J., \& Tenke, C. E. (2006). Principal components analysis of Laplacian waveforms as a generic method for identifying ERP generator patterns: II. Adequacy of lowdensity estimates. Clinical Neurophysiology, 117(2), 369-380. https://doi.org/10. 1016/j.clinph.2005.08.033.

Kok, A. (1999). Varieties of inhibition: Manifestations in cognition, event-related potentials and aging. Acta Psychologica, 101(2-3), 129-158. Retrieved from http:// www.ncbi.nlm.nih.gov/pubmed/10344183.

Kropotov, J. D., Ponomarev, V. A., Hollup, S., \& Mueller, A. (2011). Dissociating action inhibition, conflict monitoring and sensory mismatch into independent components of event related potentials in GO/NOGO task. NeuroImage, 57(2), 565-575. https:// doi.org/10.1016/J.NEUROIMAGE.2011.04.060.

Logan, G. D., Cowan, W. B., \& Davis, K. A. (1984). On the ability to inhibit simple and choice reaction time responses: A model and a method. Journal of Experimental Psychology. Human Perception and Performance, 10(2), 276-291. Retrieved from http://www.ncbi.nlm.nih.gov/pubmed/6232345.

Logan, G. D., Schachar, R. J., \& Tannock, R. (1997). Impulsivity and inhibitory control. Psychological Science, 8(1), 60-64. https://doi.org/10.1111/j.1467-9280.1997. tb00545.x.

Morein-Zamir, S., \& Meiran, N. (2003). Individual stopping times and cognitive control: Converging evidence for the stop signal task from a continuous tracking paradigm. The Quarterly Journal of Experimental Psychology. A, Human Experimental Psychology, 56(3), 469-489. https://doi.org/10.1080/02724980244000495.

Müller-Gethmann, H., Ulrich, R., \& Rinkenauer, G. (2003). Locus of the effect of temporal preparation: Evidence from the lateralized readiness potential. Psychophysiology, 40(4), 597-611. Retrieved from http://www.ncbi.nlm.nih.gov/pubmed/14570167.

Ramautar, J. R., Kok, A., \& Ridderinkhof, K. R. (2006). Effects of stop-signal modality on the N2/P3 complex elicited in the stop-signal paradigm. Biological Psychology, 72(1), 96-109. https://doi.org/10.1016/j.biopsycho.2005.08.001.
Randall, W. M., \& Smith, J. L. (2011). Conflict and inhibition in the cued-Go/NoGo task. Clinical Neurophysiology, 122(12), 2400-2407. https://doi.org/10.1016/J.CLINPH. 2011.05.012.

Ridderinkhof, K. R., van den Wildenberg, W. P. M., Segalowitz, S. J., \& Carter, C. S. (2004). Neurocognitive mechanisms of cognitive control: The role of prefrontal cortex in action selection, response inhibition, performance monitoring, and rewardbased learning. Brain and Cognition, 56(2), 129-140. https://doi.org/10.1016/j. bandc.2004.09.016.

Schevernels, H., Bombeke, K., Van der Borght, L., Hopf, J. M., Krebs, R. M., \& Boehler, C. N. (2015). Electrophysiological evidence for the involvement of proactive and reactive control in a rewarded stop-signal task. NeuroImage, 121, 115-125.

Senderecka, M. (2016). Threatening visual stimuli influence response inhibition and error monitoring: An event-related potential study. Biological Psychology, 113, 24-36.

Senderecka, M. (2017). Aversive auditory stimuli improve response inhibition and error monitoring: An event-related potential. Psychophysiology, 54, S84.

Smith, J. L., Johnstone, S. J., \& Barry, R. J. (2007). Response priming in the Go/NoGo task: The N2 reflects neither inhibition nor conflict. Clinical Neurophysiology, 118(2), 343-355. https://doi.org/10.1016/J.CLINPH.2006.09.027.

Swick, D., Ashley, V., \& Turken, U. (2011). Are the neural correlates of stopping and not going identical? Quantitative meta-analysis of two response inhibition tasks. NeuroImage, 56(3), 1655-1665. https://doi.org/10.1016/j.neuroimage.2011.02.070.

van Boxtel, G. J., van der Molen, M. W., Jennings, J. R., \& Brunia, C. H. (2001). A psychophysiological analysis of inhibitory motor control in the stop-signal paradigm. Biological Psychology, 58(3), 229-262.

van der Molen, M. W., Bashore, T. R., Halliday, R., \& Callaway, E. (1991). Chronopsychophysiology: Mental chronometry augmented by psychophysiological time markers. In J. R. Jennings, \& M. G. H. Coles (Eds.). Handbook of cognitive psychophysiology: Central and autonomic nervous system approaches (pp. 9-178). Oxford, England: John Wiley Wiley psyc.

van der Schoot, M., Licht, R., Horsley, T. M., \& Sergeant, J. A. (2005). Effects of stop signal modality, stop signal intensity and tracking method on inhibitory performance as determined by use of the stop signal paradigm. Scandinavian Journal of Psychology, 46(4), 331-341. https://doi.org/10.1111/j.1467-9450.2005.00463.x.

Verbruggen, F., \& Logan, G. D. (2008). Response inhibition in the stop-signal paradigm. Trends in Cognitive Sciences, 12(11), 418-424. https://doi.org/10.1016/j.tics.2008. 07.005 .

Verbruggen, F., \& Logan, G. D. (2009). Models of response inhibition in the stop-signal and stop-change paradigms. Neuroscience and Biobehavioral Reviews, 33(5), 647-661.

Wessel, J. R. (2017). Prepotent motor activity and inhibitory control demands in different variants of the go/no-go paradigm. Psychophysiology. https://doi.org/10.1111/psyp. 12871.

Wessel, J. R., \& Aron, A. R. (2014). Inhibitory motor control based on complex stopping goals relies on the same brain network as simple stopping. NeuroImage, 103, 225-234.

Wessel, J. R., \& Aron, A. R. (2015). It's not too late: The onset of the frontocentral P3 indexes successful response inhibition in the stop-signal paradigm. Psychophysiology, 52(4), 472-480. https://doi.org/10.1111/psyp.12374.

Wickens, C. D. (1980). The structure of attentional resources. Attention and performance VIII. Hillsdale, NY: Lawrence Erlbaum Associates239-257. 